\title{
On Approximation Algorithms for Data Mining Applications
}

\author{
Foto N. Afrati* \\ National Technical University of Athens, Greece
}

June 3, 2004

\begin{abstract}
We aim to present current trends in the theoretical computer science research on topics which have applications in data mining. We briefly describe data mining tasks in various application contexts. We give an overview of some of the questions and algorithmic issues that are of concern when mining huge amounts of data that do not fit in main memory.
\end{abstract}

\section{Introduction}

Data mining is about extracting useful information from massive data such as finding frequently occurring patterns or finding similar regions or clustering the data. From the algorithmic point of view mining algorithms seek to compute good approximate solutions to the problem at hand. As a consequence of the huge size of the input, algorithms are usually restricted to making only a few passes over the data, and they have limitations on the random access memory they use and the time spent per data item. The advent of the internet has added new applications and challenges to this area too.

The input in a data mining task can be viewed, in most cases, as a two dimensional $m \times n$ 0,1-matrix which often is sparse. This matrix may represent several objects such as a collection of documents (each row is a document and each column is a word and there is a 1 entry if the word appears in this document), or a collection of retail records (each row is a transaction record and each column represents an item, there is a 1 entry if the item was bought in this transaction), or both rows and columns are sites on the web and there is a 1 entry if there is a link from the one site to the other. In the latter case, the matrix is often viewed as a graph too. Sometimes the matrix can be viewed as a sequence of vectors (its rows) or even a sequence of vectors with integer values (not only 0,1 ).

${ }^{*}$ This work was partially supported by European Commission, Project APOLL-IST-1999-14084 and the Greek General Secretariat of Research and Technology, Project on Data Mining. 
The performance of a data mining algorithm is measured in terms of the number of passes, the required work space in main memory and computation time per data item. A constant number of passes is acceptable but one pass algorithms are mostly sought for. The workspace available ideally is constant but sublinear space algorithms are also considered. The quality of the output is usually measured using conventional approximation ratio measures [97], although in some problems the notion of approximation and the manner of evaluating the results remain to be further investigated. Space and time per item is measured as a function of $N$, the number of items read by the algorithm.

These performance constraints call for designing novel techniques and novel computational paradigms. Since the amount of data far exceeds the amount of workspace available to the algorithm, it is not possible for the algorithm to "remember" large amounts of past data. A recent approach is to create a summary of the past data to store in main memory, leaving also enough memory for the processing of the future data. Using a random sample of the data is also another popular technique.

Besides data mining, other applications can be also modeled as one pass problems such as the interface between the storage manager and the application layer of a database system or processing data that are brought to desktop from networks, where each pass essentially is another expensive access to the network. Several communities have contributed (with technical tools and methods as well as by solving similar problems) to the evolving of the data mining field, including statistics, machine learning and databases.

Many single pass algorithms have been developed recently and also techniques and tools that facilitate them. We will review some of them here. In the first part of this chapter (next two sections), we review formalisms and technical tools used to find solutions to problems in this area. In the rest of the chapter we briefly discuss recent research in association rules, clustering and web mining. An association rule relates two columns of the entry matrix (e.g., if the $i$-th entry of a row $v$ is 1 then most probably the $j$-th entry of $v$ is also 1 ). Clustering the rows of the matrix according to various similarity criteria in a single pass is a new challenge which traditional clustering algorithms did not have. In web mining, one problem of interest in search engines is to rank the pages of the web according to their importance on a topic. Citation importance is taken by popular search engines according to which important pages are assumed to be those that are linked by other important pages.

In more detail the rest of the chapter is organized as follows. The next section contains formal techniques used for single pass algorithms and a formalism for the data stream model. Section 3 contains an algorithm with performance guarantees for finding approximately the $L_{p}$ distance between two data streams. Section 4 contains a list of what are considered the main data mining tasks and another list with applications of these tasks. The last three sections discuss recent algorithms developed for finding association rules, clustering a set of data items and for searching the web for useful information. In these three sections, techniques mentioned in the beginning of the chapter are used (such as SVD, sampling) to solve the specific problems. Naturally some of the techniques are common, such as, for example, spectral methods are used in both clustering and web mining. 


\section{Formal Techniques and Tools}

In this section we present some theoretical results and formalisms that are often used in developing algorithms for data mining applications. In this context, the singular value decomposition (SVD) of a matrix (subsection 2.1.2) has inspired web search techniques, and, as a dimensionality reduction technique, is used for finding similarities among documents or clustering documents (known as the latent semantic indexing technique for document analysis). Random projections (subsection 2.1.1) offer another means for dimensionality reduction explored in recent work. Data streams (subsection 2.2) is proposed for modeling limited pass algorithms; in this subsection some discussion is done on lower and upper bounds on the required workspace. Sampling techniques (subsection 2.3) have also been used in statistics and learning theory, under somewhat different perspective however. Storing a sample of the data that fits in main memory and running a "conventional" algorithm on this sample is often used as the first stage of various data mining algorithms. We discuss the problem of selecting a random sample out of a dataset. We present a computational model for probabilistic sampling algorithms that compute approximate solutions. This model is based on the decision tree model [27] and relates the query complexity to the size of the sample.

We start by providing some (mostly) textbook definitions for self containment purposes. In data mining we are interested in vectors and their relationships under several distance measures. For two vectors, $\vec{v}=\left(v_{1}, \ldots, v_{n}\right), \quad \vec{u}=\left(u_{1}, \ldots, u_{n}\right)$, the dot product or inner product is defined to be a number which is equal to the sum of the component-wise products $\vec{v} \cdot \vec{u}=v_{1} u_{1}+\ldots+v_{n} u_{n}$ and the $L_{p}$ distance (or $L_{p}$ norm) is defined to be: $\|\vec{v}-\vec{u}\|_{p}=\left(\sum_{i=1}^{n}\left|v_{i}-u_{i}\right|^{p}\right)^{1 / p}$. The $L_{p}$ distance is extended to be defined between matrices : $\|\vec{V}-\vec{U}\|_{p}=\left(\Sigma_{i}\left(\Sigma_{j}\left|V_{i j}-U_{i j}\right|^{p}\right)\right)^{1 / p}$. We sometimes use \|\| to denote \|\|$_{2}$. The cosine distance is defined to be $1-\frac{\vec{v} \cdot \vec{u}}{\|\vec{v}\|}\|\vec{u}\|$. For sparse matrices the cosine distance is a suitable similarity measure as the dot product deals only with non-zero entries (which are the entries that contain the information) and then it is normalized over the lengths of the vectors.

Given a sequence of reals $x_{1}, \ldots, x_{n}$, the $k$-th statistical moment is defined as $\mu_{k}\left(x_{1}, \ldots, x_{n}\right)=1 / n \sum_{i=1}^{n} x_{i}^{k}$. The first statistical moment is simply the mean. Some results are based on stable distributions [85]. A distribution $\mathcal{D}$ over the reals is called $p$-stable if for any $n$ real numbers $a_{1}, \ldots, a_{n}$ and independent identically distributed, with distribution $\mathcal{D}$, variables $X_{1}, \ldots, X_{n}$, the random variable $\Sigma_{i} a_{i} X_{i}$ has the same distribution as the variable $\left(\Sigma_{i}\left|a_{i}\right|^{p}\right)^{1 / p} X$, where $X$ is a random variable with the same distribution as the variables $X_{1}, \ldots, X_{n}$. It is known that stable distributions exist for any $p \in(0,2]$. A Cauchy distribution defined by the density function $\frac{1}{\pi\left(1+x^{2}\right)}$, is

1-stable, a Gaussian (normal) distribution defined by the density function $\frac{1}{\sqrt{2 \pi}} e^{-x^{2} / 2}$, is 2-stable.

A randomized algorithm [81] is an algorithm that flips coins, i.e., it uses random bits, while no probabilistic assumption is made on the distribution of the input. A randomized algorithm is called Las-Vegas if it gives the correct answer on all inputs. Its running time or workspace could be a random variable depending on the random variable of the coin tosses. A randomized algorithm is called Monte-Carlo with error probability $\epsilon$ if on every input it gives the right answer with probability at least $1-\epsilon$. 


\subsection{Dimensionality Reduction}

Given a set $S$ of points in the multidimensional space, dimensionality reduction techniques are used to map $S$ to a set $S^{\prime}$ of points in a space of much smaller dimensionality while approximately preserving important properties of the points in $S$. Usually we want to preserve distances. Dimensionality Reduction techniques can be useful in many problems where distance computations and comparisons are needed. In high dimensions distance computations are very slow and moreover it is known that, in this case, the distance between almost all pairs of points is the same with high probability and almost all pair of points are orthogonal (known as the Curse of Dimensionality).

Dimensionality reduction techniques that are popular recently include Random Projections and Singular Value Decomposition (SVD). Other dimensionality reduction techniques use linear transformations such as the Discrete Cosine transform or Haar Wavelet coefficients or the Discrete Fourier Transform (DFT). DFT is a heuristic which is based on the observation that, for many sequences, most of the energy of the signal is concentrated in the first few components of DFT. The $L_{2}$ distance is preserved exactly under the DFT and its implementation is also practically efficient due to an $O(n \log n)$ DFT algorithm. Dimensionality reduction techniques are well explored in databases [51, 43].

\subsubsection{Random Projections}

Random Projection techniques are based on the Johnson-Lindenstrauss (JL) lemma [67] which states that any set of $n$ points can be embedded into the $k$-dimensional space with $k=O\left(\log n / \epsilon^{2}\right)$ so that the distances are preserved within a factor of $\epsilon$.

Lemma 2.1 (JL) Let $\vec{v}_{1}, \ldots, \vec{v}_{m}$ be a sequence of points in the d-dimensional space over the reals and let $\epsilon, F \in(0,1]$. Then there exists a linear mapping $f$ from the points of the d-dimensional space into the points of the $k$-dimensional space where $k=O\left(\log (1 / F) / \epsilon^{2}\right)$ such that the number of vectors which approximately preserve their length is at least $(1-F) m$. We say that a vector $\vec{v}_{i}$ approximately preserves its length if:

$$
\left\|\vec{v}_{i}\right\|^{2} \leq\left\|f\left(\vec{v}_{i}\right)\right\|^{2} \leq(1+\epsilon)\left\|\vec{v}_{i}\right\|^{2}
$$

The proof of the lemma, however, is non-constructive: it shows that a random mapping induces small distortions with high probability. Several versions of the proof exist in the literature. We sketch the proof from [65]. Since the mapping is linear, we can assume without loss of generality that the $\vec{v}_{i}$ 's are unit vectors. The linear mapping $f$ is given by a $k \times d$ matrix $\vec{A}$ and $f\left(\vec{v}_{i}\right)=\vec{A} \vec{v}_{i}, i=1, \ldots, m$. By choosing the matrix $\vec{A}$ at random such that each of its coordinates is chosen independently from $N(0,1)$, then each coordinate of $f\left(\vec{v}_{i}\right)$ is also distributed according to $N(0,1)$ (this is a consequence of the spherical symmetry of the normal distribution). Therefore, for any vector $\vec{v}$, for each $j=1, \ldots, k / 2$, the sum of squares of consecutive coordinates $Y_{j}=\left\|f(\vec{v})_{2 j-1}\right\|^{2}+\left\|f(\vec{v})_{2 j}\right\|^{2}$ has exponential distribution with exponent $1 / 2$. The expectation of $L=\|f(\vec{v})\|^{2}$ is equal to $\Sigma_{j} E\left[Y_{j}\right]=k$. It can be shown that the value of $L$ lies within $\epsilon$ of its mean with probability $1-F$. Thus the expected number of vectors whose length is approximately preserved is $(1-F) m$. 
The JL lemma has been proven useful in improving substantially many approximation algorithms (e.g., [65, 17]). Recently in [40], a deterministic algorithm is presented which finds such mapping in time almost linear in the number of distances to preserve times the dimension $d$ of the original space. In recent work, random projections are used to compute summaries of past data called sketches to solve problems such as approximating the $L_{p}$ norm of a data stream (see also section 3 ).

\subsubsection{Singular Value Decomposition}

Consider matrices with real numbers as entries. We say that a matrix $\vec{M}$ is orthogonal if $\vec{M} \vec{M}^{T r}=\vec{I}$ where $\vec{I}$ is the identity matrix (by $\vec{A}^{T r}$ we denote the transpose of matrix $\vec{A})$. An eigenvalue of a $n \times n$ matrix $\vec{M}$ is a number $\lambda$ such that there is a vector $\vec{t}$ which satisfies $\vec{M} \vec{t}=\lambda \vec{t}$. Such a vector $\vec{t}$ is called an eigenvector associated with $\lambda$. The set of all eigenvectors associated with $\lambda$ form a subspace and the dimension of this subspace is called the multiplicity of $\lambda$. If $\vec{M}$ is a symmetric matrix, then the multiplicities of all eigenvalues sum up to $n$. Let us denote all the eigenvalues of such a matrix $\vec{M}$ by $\lambda_{1}(\vec{M}), \lambda_{2}(\vec{M}), \ldots \lambda_{n}(\vec{M})$, where we have listed each eigenvalue a number of times equal to its multiplicity. For symmetric matrix $\vec{M}$, we can choose for each $\lambda_{i}(\vec{M})$ an associated eigenvector $\vec{t}_{i}(\vec{M})$ such that the set of vectors $\left\{\vec{t}_{i}(\vec{M})\right\}$ forms an orthonormal basis for the $n$-dimensional space over the real numbers. Let $\vec{Q}$ be the matrix with columns these vectors and let $\Lambda$ be the diagonal matrix with diagonal entries the list of eigenvalues. Then, it is easy to prove that: $\vec{M}=\vec{Q} \Lambda \vec{Q}^{T r}$. However the result extends to any matrix as the following theorem states.

Theorem 2.1 (Singular Value Decomposition/SVD) Every $m \times n$ matrix $\vec{A}$ can be written as $\vec{A}=\vec{U} \vec{T} \vec{V}^{T r}$ where $\vec{U}$ and $\vec{V}$ are orthogonal and $\vec{T}$ is diagonal.

The diagonal entries of $\vec{T}$ are called the singular values of $\vec{A}$. It is easy to verify that the columns of $\vec{U}$ and $\vec{V}$ represent the eigenvectors of $\vec{A} \vec{A}^{T r}$ and $\vec{A}^{T r} \vec{A}$ respectively and the diagonal entries of $\vec{T}^{2}$ represent their common set of eigenvalues. The importance of the SVD in dimensionality reduction lies in the following theorem which states that $\vec{U}, \vec{T}, \vec{V}$ can be used to compute, for any $k$, the matrix $A_{k}$ of rank $k$ which is "closest" to $\vec{A}$ over all matrices of rank $k$.

Theorem 2.2 Let the $S V D$ of $\vec{A}$ be given by $\vec{A}=\vec{U} \vec{T} \vec{V}^{T r}$. Suppose $\tau_{1}, \ldots, \tau_{k}$ are the $k$ largest singular values. Let $\vec{u}_{i}$ be the $i$-th column of $\vec{U}$ and $\vec{v}_{i}$ be the $i$-th column of $\vec{V}$ and let $\tau_{i}$ be the $i$-th element in the diagonal of $\vec{T}$. Let $r$ be the rank of $\vec{A}$ and let $k<r$. If

$$
\vec{A}_{k}=\sum_{i=1}^{k} \tau_{i} \vec{u}_{i} \vec{v}_{i}^{T r}
$$

Then

$$
\min _{\operatorname{rank}(\vec{B})=k}\|\vec{A}-\vec{B}\|_{2}=\left\|\vec{A}-\vec{A}_{k}\right\|_{2}=\tau_{k+1}
$$

The SVD technique displays optimal dimensionality reduction (for linear projections) but it is hard to compute. 


\subsection{The Data Stream Computation Model}

The streaming model is developed to formalize a single (or few) pass(es) algorithm over massive data that do not fit in main memory. In this model, the data is observed once (or few times) and in the same order it is generated. For each data item, we want to minimize the required workspace and the time to process it.

In the interesting work of [61] where the stream model was formalized, a data stream is defined as a sequence of data items $v_{1}, v_{2}, \ldots, v_{n}$ which are assumed to be read by an algorithm only once (or very few times) in increasing order of the indices $i$. The number $P$ of passes over the data stream and the workspace $W$ (in bits) required by the algorithm in the main memory are measured. The performance of an algorithm is measured by the number of passes the algorithm makes over the data and the required workspace, along with other measures such as the computation time per input data item. This model does not necessarily require a bound on the computation time.

Tools from communication complexity are used to show lower bounds on the workspace of limited-pass algorithms [8],[61]. Communication complexity [79] is defined as follows. In the (2-party) communication model there are two players $A$ and $B$. Player $A$ is given a $x$ from a finite set $X$ and player $B$ is given a $y$ from a finite set $Y$. They want to compute a function $f(x, y)$. As player $A$ does not know $y$ and player $B$ does not know $x$, they need to communicate. They use a protocol to exchange bits. The communication complexity of a function $f$ is the minimum over all communication protocols of the maximum over all $x \in X, y \in Y$ of the number of bits that need to be exchanged to compute $f(x, y)$. The protocol can be deterministic, Las-Vegas or Monte-Carlo. If one player is only transmitting and one is only receiving then it is called one-way communication complexity. In this case, only the receiver needs to be able to compute function $f$.

To see how communication complexity is related to deriving lower bounds on the space, think of one way communication where player $A$ has the information of the past data and player $B$ has the information of the future data. The communication complexity can be used as a lower bound on the space available to store a "summary" of the past data.

It is natural to ask whether under the stream model there are noticeable differences regarding the workspace requirements (i) between one-pass and multi-pass algorithms, (ii) between deterministic and randomized algorithms and (iii) between exact and approximation algorithms. These questions were explored in earlier work [82] in context similar to data streams and it was shown that: (i) Some problems require a large space in one pass and a small space in two passes. (ii) There can be an exponential gap in space bounds between Monte-Carlo and Las-Vegas algorithms. (iii) For some problems, an algorithm for an approximate solution, requires substantially less space than an exact solution algorithm.

In [8], space complexity for estimating the frequency moments of a sequence of elements in one pass was studied and tight lower bounds were derived. The problem studied in [82] is the space required for selecting the $k$-th largest out of $n$ elements using at most $P$ passes over the data. An upper bound of $n^{1 / P} \log n$ and a lower bound of $n^{1 / P}$ is shown, for large enough $k$. Recent work on space lower bounds includes also [90].

The data stream model appears to be related to other work e.g., on competitive analysis [69], or I/O efficient algorithms [98]. However, it is more restricted in that it 
requires that a data item can never again be retrieved in main memory after its first pass (if it is a one-pass algorithm). A distributed stream model is also proposed in [53] which combines features of both streaming models and communication complexity models.

Streaming models have been extensively studied recently and methods have been developed for comparing data streams under various $L_{p}$ distances, or clustering them. The stream model from the database perspective is investigated in the Stanford stream data management Project [93] (see [11] for an overview and algorithmic considerations).

\subsection{Sampling}

Randomly sampling a few data items of a large data input is often a technique used to extract useful information about the data. A small sample of the data may be sufficient to compute many statistical parameters of the data with reasonable accuracy. Tail inequalities from probability theory and the central limit theorem are useful here [81, 47].

One of the basic problems in this context is to computes the size of the sample required to determine certain statistical parameters. In many settings, the size of the sample for estimating the number of distinct values in a data set is of interest. The following proposition [86] gives a lower bound on the size of the sample in such a case whenever we know the number of distinct values and each has a frequency greater than $\epsilon$.

Proposition 2.1 If a dataset $D$ contains $l \geq k$ distinct values of frequency at least $\epsilon$, then a sample of size $s \geq \frac{1}{\epsilon} \log \frac{k}{\delta}$ contains at least $k$ distinct values with probability $>1-\delta$.

To prove, let $a_{1}, \ldots, a_{l}$ be the $l$ distinct values of frequencies $p_{1}, \ldots, p_{l}$ respectively and, each frequency is at least $\epsilon$. Then the probability our sample missed $k$ of these distinct values is at most $\sum_{i=1}^{k}\left(1-p_{i}\right)^{s} \leq k(1-\epsilon)^{s} \leq \delta$ by our choice of $s$.

In a similar context, random sampling from a dataset whose size is unknown, is of interest in many applications. The problem is to select a random sample of size $n$ from a dataset of size $N$ when $N$ is unknown. A one-pass reservoir algorithm is developed in [99]. A reservoir algorithm maintains a sample (reservoir) of data items in main memory and data items may be selected for the reservoir as they are processed. The final random sample will be selected from the sample maintained in the reservoir (hence the size of the sample in the reservoir is larger than $n$ ). In [99] each data item is selected with probability $M / n$ where $n$ is the number of data items read so far and $M$ is the size of the reservoir.

An algorithm that uses a sample of the input is formalized in [14] as a uniform randomized decision tree. This formalism is used to derive lower bounds on the required size of the sample. A randomized decision tree has two kinds of internal nodes, query nodes and random coin toss nodes. Leaves are related to output values. On an input $x_{1}, \ldots, x_{n}$, the computation of the output is done by following a path from the root to a leaf. On each internal node a decision is made as to which of its children the computation path moves next. In a random coin toss node this decision is based on a coin toss which picks one of the children uniformly at random. A query node $v$ has two labels, an input location (to be queried), and a function which maps a sequence of 
query locations (the sequence is thought of as the input values queried so far along the path from the root) to one of the children of this node $v$. The child to which the path moves next is specified by the value of this function. Each leaf is labeled by a function which maps the sequence of query locations read along the path to an output value. The output is the value given by the function on the leaf which is the end point of the computation path.

Note that any input $x_{1}, \ldots, x_{n}$ may be associated with several possible paths leading from the root to a leaf, depending on the random choices made in the random coin nodes. These random choices induce a distribution over the paths corresponding to $x_{1}, \ldots, x_{n}$.

A uniform randomized decision tree is defined as a randomized decision tree with the difference that each query node is not labeled by an input variable. The query in this case is done uniformly at random over the set of input values that have not been queried so far along the path from the root. A uniform decision tree can be thought as a sampling algorithm which samples the input uniformally at random and uses only these sample values to decide the output. Thus the number of query nodes along a path from the root to a leaf is related to the size of the sample.

The expected query complexity of a decision tree $T$ on input $\vec{x}=x_{1}, \ldots, x_{n}$ denoted $S^{e}(T, \vec{x})$, is the expected number of query nodes on paths corresponding to $\vec{x}$. The worst case query complexity of a tree $T$ on input $\vec{x}$, denoted $S^{w}(T, \vec{x})$, is the maximum number of query nodes on paths corresponding to $\vec{x}$. Here the expectation and the maximum are taken over the distribution of paths.

The expected and worst case query complexity of $T S^{e}(T)$ and $S^{w}(T)$ are the maximum of $S^{e}(T, \vec{x})$ and $S^{w}(T, \vec{x})$, respectively, over all inputs $\vec{x}$ in $A^{n}$.

Because of the relation between query complexity and the size of the required sample, a relationship can also be obtained between query complexity and space complexity as defined in the data stream model. Let $\epsilon \geq 0$ be an error parameter, $\delta(0<\delta<1)$ a confidence parameter, and $f$ a function. A decision tree is said to $(\epsilon, \delta)$-approximate $f$ if for every input $\vec{x}$ the probability of paths corresponding to $\vec{x}$ that output a values $y$ within a factor of $\epsilon$ from the exact solution is at least $1-\delta$. The $(\epsilon, \delta)$ expected query complexity of $f$ is:

$$
S_{\epsilon, \delta}^{e}(f)=\min \left\{S^{e}(T) \mid T \quad(\epsilon, \delta) \text { - approximates } f\right\}
$$

The worst case query complexity of a function $f$ is defined similarly.

The $(\epsilon, \delta)$ query complexity of a function $f$ can be directly related to the space complexity as defined on data streams. If a function has $(\epsilon, \delta)$ query complexity $S_{\epsilon, \delta}^{e}(f)$, then the space required in main memory is at most $S_{\epsilon, \delta}^{w}(f) O(\log |A|+\log n)$, where $A, n$ are parameters of the input vector $\vec{x}$. For input vector $\vec{x}=x_{1}, \ldots, x_{n}, n$ is the number of data items and $A$ is the number of elements from which the values of each $x_{i}$ is drawn.

Based on this formalization, a lower bound is obtained on the number of samples required to distinguish between two distributions [14]. It it also shown that the $k$-th statistical moment can be approximated within an additive error of $\epsilon$ by using a random sample of size $O\left(1 / \epsilon^{2} \log \frac{1}{\delta}\right)$, and that this is a lower bound on the size of the sample. Work that also refer to lower bounds on query complexity for approximate solutions include results on the approximation of the mean [28], [36, 91], the approximation on the frequency moment [31]. 
Lossy compression may be related to sampling. When we have files in compressed form, we might want to compute functions of the uncompressed file without having to decompress. Compressed files might even been thought of as not been able to be precisely retrieved by decompression, namely the compression (in order to gain larger compression factors) allowed for some loss of information (lossy compression). Problems of this nature are related to sampling algorithms in [46].

Statistical decision theory and statistical learning theory are fields where sampling methods are used too. However they focus on different issues than data mining does. Statistical decision theory [16] studies the process of making decisions based on information gained by computing various parameters of a sample. However the sample is assumed given and methods are developed that maximize the utitily of it. Computing the required size of a sample for approximately computing parameters of the input data is not one of its concerns. Statistical learning theory $[96,70]$ is concerned with learning an unknown function from a class of target functions, i.e., approximating the function rather, whereas, in data mining, the interest is in approximating some parameter of the function.

For an excellent overview on key research results and formal techniques on data stream algorithms see the tutorial in [51] and references therein. Also an excellent survey on low distortion embedding techniques for dimensionality reduction can be found in [63].

\section{Approximating the $L_{p}$ distance. Sketches.}

We consider in this section the following problem which may be part of various data mining tasks. The data stream model is assumed and we want to compute an approximation to the $L_{p}$ distance. Formally, we are given a stream $S$ of data items. Each data item is viewed as a pair $(i, v), i=1, \ldots, n$, with entries for $v$ an integer in the range $\{-M, M\}$ where $M$ is a positive integer (so we need $\log M$ memory to store the value of each data item). Note that there may exist several pairs (with possibly different values for $v$ ) for a specific $i$. We want to compute a good approximation of the following quantity:

$$
L_{p}(S)=\left(\Sigma_{i=1, \ldots, n}\left|\Sigma_{(i, v) \in S} v\right|^{p}\right)^{1 / p}
$$

The obvious solution to this problem, i.e., maintain a counter for each $i$ is too costly because of the size of the data. In the influential paper [8], a scheme is proposed for approximating $L_{2}(S)$ within a factor of $\epsilon$ in workspace $O(1 / \epsilon)$ with arbitrarily large constant probability.

In [46], a solution for $L_{1}(S)$ is investigated for the special case where there are at most two non zero entries for each $i$. In this case, the problem can be equivalently viewed as having two streams $S_{a}$ and $S_{b}$ and asking for a good approximation of $L_{1}\left(S_{a}, S_{b}\right)=\Sigma_{i}\left|\Sigma_{(i, v) \in S_{a}} v-\Sigma_{(i, v) v \in S_{b}} v\right|$. A single pass algorithm is developed which, with probability $1-\delta$, computes an approximation to $L_{1}(S)$ within a factor of $\epsilon$ using $O\left(\log M \log n \log (1 / \delta) / \epsilon^{2}\right)$ random access space and $O(\log n \log \log n+$ $\left.\log M \log (1 / \delta) / \epsilon^{2}\right)$ computation time per item. The method in [46] can be viewed as 
using sketches of vectors, which is a summary data structure. In this case, a sketch $C\left(S_{a}\right), C\left(S_{b}\right)$ is computed for each data stream $S_{a}, S_{b}$ respectively. Sketches are much smaller in size than $S_{a}, S_{b}$ and such that an easily computable function of the sketches gives a good approximation to $L_{1}\left(S_{a}, S_{b}\right)$.

In [62], a unifying framework is proposed for approximating $L_{1}(S)$ and $L_{2}(S)$ within a factor of $\epsilon$ (with probability $1-\delta$ ) using $O\left(\log M \log (n / \delta) \log (1 / \delta) / \epsilon^{2}\right)$ random access space and $O(\log (n / \delta))$ computation time per item. The technique used combines the use of stable distributions [85] with Nisan pseudorandom generators [84]. The property of stable distributions which is used in this algorithm is the following. The dot product of a vector $\vec{u}$ with a sequence of $n$ independent identically distributed random variables having $p$-stable distribution is a good estimator of the $L_{p}$ norm of $\vec{u}$. In particular we can use several such products to embed a $d$-dimensional space into some other space (of lower dimensionality) such that to approximately preserve the $L_{p}$ distances. Dot product can be computed in small workspace.

We shall describe here in some detail the first stage of this algorithm for approximating $L_{1}(S)$ : For $l=O\left(c / \epsilon^{2} \log 1 / \delta\right.$ ) (for some suitable constant $c$ ), we initialize $n l$ independent random variables $X_{i}^{j}, i=1, \ldots, n, j=1, \ldots, l$ with Cauchy distribution defined by the density function $f(x)=\frac{1}{\pi} \frac{1}{1+x^{2}}$ (we know this distribution is 1-stable). Then, the following three steps are executed:

1. Set $S^{j}=0$, for $j=1, \ldots, l$.

2. For each new pair $(i, v)$ do: $S^{j}=S^{j}+v X_{i}^{j}$ for all $j=1, \ldots, l$.

3. Return the median $\left(\left|S^{0}\right|, \ldots,\left|S^{l-1}\right|\right)$.

To prove the correctness of this algorithm we argue as follows: We want to compute $L_{1}(S)=C=\Sigma_{i}\left|c_{i}\right|$ where $c_{i}=\Sigma_{(i, v) \in S} v$. First, it follows from the 1-stability of the Cauchy distribution that, each $S^{j}$ has the same distribution as $C X$ where $X$ has Cauchy distribution. Random variable $X$ has Cauchy distribution with density function $f(x)=\frac{1}{\pi} \frac{1}{1+x^{2}}$ hence median $(|X|)=1$ and median $(v|X|)=v$ for any $v$. It is known that for any distribution, if we take $l=O\left(c / \epsilon^{2} \log 1 / \delta\right)$ independent samples and compute the median $M$, then for distribution function $F(M)$ of $M$ we have (for a suitable constant $c) \operatorname{Pr}[F(X) \in[1 / 2-\epsilon, 1 / 2+\epsilon]]>1-\delta$. Thus, it can be proven that $l=O\left(c / \epsilon^{2} \log 1 / \delta\right)$ independent samples approximate $L_{1}(S)$ within a factor of $\epsilon$ with probability $>1-\delta$.

This stage of the algorithm, though, assumes random numbers of exact precision. Thus, random generators are used to solve the problem of how to reduce the number of required random bits.

The problem of approximating $L_{p}$ distances in one pass algorithms has a variety of applications including estimation of the size of self join $[8,52]$ and estimation of statistics of network flow data [46].

In the above frameworks a solution was facilitated by using summary descriptions of the data which approximately preserved the $L_{p}$ distances. The summaries were based on computing with random variables. Techniques that use such summaries to reduce the size of the input are known as sketching techniques (they compute a sketch of each 
input vector). Computing sketches has been used with success in many problems to get summaries of the data. It has enabled compression of data and has been speeding up computation for various data mining tasks [64, 34, 25, 26, 35]. (See also section 5 for a description of the algorithm in [34].) Sketches based on random projections are often used to approximate $L_{p}$ distances or other measure of similarities depending on them. In such a case (see e.g., [35]) sketches are defined as: The $i$-th component of the sketch $\vec{s}(\vec{x})$ of $\vec{x}$ is the dot product of $\vec{x}$ with a random vector $\vec{r}_{i}$.

$$
\vec{s}_{i}(\vec{x})=\vec{x} \quad \cdot \quad \vec{r}_{i}
$$

where each component of each random vector is drawn from a Cauchy distribution.

Work that use sketching techniques include $[38,49]$ where aggregate queries and multi-queries over data streams are computed.

\section{Data Mining Tasks and Applications}

The data available for mining interesting knowledge (e.g., census data, corporate data, biological data) is often in bad shape (have been gathered under no particular considerations), e.g. it may contain duplicate or incomprehensible information. Therefore a preprocessing stage is required to clean the data. The main data mining tasks are considered to be those that have an almost well defined algorithmic objective and assume that the given data are in a good shape. Moreover after the results of a data mining task are obtained they may need a post processing stage to interprete and visualize them. In this section, we mention some areas of research and applications that are considered of interest in the data mining community [59]. We begin with a list of the most common data mining tasks:

- Association rules: Find correlations among the columns of the input matrix of the form: if there is a 1 entry in column 5 then most probably there is a 1 entry in column 7 too. These rules are probabilistic in nature.

- Sequential patterns: Find sequential patterns that occur often in a dataset.

- Time series similarity: Find criteria that check in a useful way whether two sequences of data exhibit "similar features".

- Sequence matching: Given a collection of sequences and a sequence query, find the sequence which is closest to the query-sequence.

- Clustering: Partition a given set of points into groups, called clusters so that "similar" points belong to the same cluster. A measure of similarity is needed, often it is a distance in a metric space.

- Classification: Given a set of points and a set of labels, assign labels to point so that similar objects are labeled by similar labels and a point is labeled by the most likely label. A measure of similarity of points and similarity of labels is assumed and a likelihood of a point to be assigned a particular label.

- Discovery of outliers: Discover points in the dataset which are isolated, i.e., they do not belong to any multi-populated cluster.

- Frequent episodes: An extension of sequential pattern finding, where more complex patterns are considered. 
These tasks as described in the list are usually decomposed in more primitive modules that may be common in several tasks, e.g., comparing large pieces of the input matrix to find similarity patterns is useful in clustering, and association rules mining.

We include a list of some of the most common applications of data mining:

- Marketing. Considered one of the most known successes of data mining. Market basket analysis is motivated by the decision support problem and aims at observing customer habits to decide on business policy regarding prices or product offers. Basket data are collected by most large retail organizations and used mostly for marketing purposes. In this context, it is of interest to discover association rules such as "if a person buys pencil then most probably buys paper too". Such information can be used to increase sales on pencils by placing them near paper or make a profit by offering good prices on pencils and increase the price of paper.

- Astronomy. Clustering celestial objects by their radiation to distinguish galaxies and other star formations.

- Biology. Correlate diabetes to the presence of certain genes. Find DNA sequences representing genomes (sequential patterns). Work in time series analysis has many practical applications here.

- Document analysis. Cluster documents by subject. Used, for example, in collaborative filtering, namely tracking user behavior and making recommendations to individuals based on similarity of their preferences to these of other users.

- Financial Applications. Use time series similarity to find stocks with similar price movements or find products with similar selling patterns. Observe similar patterns in customers' financial history to decide if a bank loan is awarded.

- Web mining. Search engines like Google rank web pages by their "importance" in order to decide the order on which to present search results on a user query. Identifying communities on the web, i.e., groups that share a common interest and have a large intersection of web pages that are most often visited by the members of a group. This may be useful for advertising or to identify the most up-to-date information on a topic or to provide a measure of page rank which is not easy to spam. One popular method is to study co-citation and linkage statistics: web communities are characterized by dense directed bipartite subgraphs.

- Communications. Discover the geographic distribution of cell phone traffic at different base stations or the evolution of traffic at Internet routers over time. Detecting similarity patterns over such data is important, e.g., which geographic regions have similar cell phone usage distribution, or which IP subnet traffic distributions over time intervals are similar.

- Detecting intrusions. Detecting intrusions the moment they happen is important to protecting a network from attack. Clustering is a technique used to detecting intrusions.

- Detecting failures in network. Mining episodes helps to detect faults in electricity network before they occur or detect congestions in packet switched networks. 


\section{$5 \quad$ Association Rules}

Identifying association rules in market basket data is considered to be one of the most well known successes of the data mining field. The problem of mining for association rules and the related problem of finding frequent itemsets have been studied extensively and many efficient heuristics are known. We will mention some of them in this section.

Basket data is a collection of records (or baskets), each record typically consisting of a transaction date and a collection of items (thought of as the items bought in this transaction). Formally we consider a domain set $\mathcal{I}=\left\{i_{1}, \ldots, i_{m}\right\}$ of elements called items and we are given a set $\mathcal{D}$ of transactions where each transaction $T$ is a subset of $\mathcal{I}$. We say that a transaction $T$ contains a set $X$ of items if $X \subseteq T$. Each transaction is usually viewed as a row in a $n \times k 0,1$-matrix where 1 means that the item represented by this column is included in this transaction and 0 that it is not included. The rows represent the baskets and the columns represent the items in the domain. The columns are sometimes called attributes or literals. Thus an instance of marker basket data is represented by a 0,1 -matrix.

The problem of mining association rules over basket data was introduced in [4]. An association rule is an "implication" rule $X \Rightarrow Y$ where $X \subset \mathcal{I}$ and $Y \subset \mathcal{I}$ and $X, Y$ are disjoint. The rule $X \Rightarrow Y$ holds in the transaction set $\mathcal{D}$ with confidence $c$ if $c \%$ of the transactions in $\mathcal{D}$ that contain $X$ also contain $Y$. The rule $X \Rightarrow Y$ has support $s$ in the transaction set $\mathcal{D}$ if $s \%$ of the transactions in $\mathcal{D}$ contain $Y \cup X$. The symbol $\Rightarrow$ used in an association rule is not a logical implication, it only denotes that the confidence and the support are estimated above the thresholds $c \%$ and $s \%$ respectively. In this context, the problem of mining for association rules on a given transaction set asks to generate all association rules with confidence and support thresholds greater than two given integers.

Functional dependencies are association rules with $100 \%$ confidence and any support and they are denoted as $X \rightarrow A$. Consequently, having determined a dependency $X \rightarrow A$, any dependency of the form $X \cup Y \rightarrow A$ can be ignored as redundant. The general case of association rules however is probabilistic in nature. Hence a rule $X \Rightarrow A$ does not make rule $X \cup Y \Rightarrow A$ redundant because the latter may not have minimum support. Similarly, rules $X \Rightarrow A$ and $A \Rightarrow Z$ do not make rule $X \Rightarrow Z$ redundant because the latter may not have minimum confidence.

In the context of the association rule problem, mining for frequent itemsets is one of the major algorithmic concerns. The frequent itemsets problem asks to find all sets of items (itemsets) that have support above a given threshold. This problem can be reduced to finding all the maximal frequent itemsets due to the monotonicity property - i.e., any subset of a frequent itemset is a frequent itemset too. A frequent itemset is maximal if any itemset which contains it is not frequent.

\subsection{Mining for frequent itemsets}

The monotonicity property has inspired a large number of algorithms known as a-priori algorithms which use the following a-priori trick: The algorithms begin the search for frequent itemsets with searching for frequent items and then construct candidate pairs of items only if both items in the pair are frequent. In the same fashion, they construct frequent candidate triples of items only if all the three pairs items in the triple are found frequent in the previous step. Thus, to find frequent itemsets, they proceed 
levelwise, finding first the frequent items (sets of size 1), then the frequent pairs, the frequent triples, and so on.

An a-priori algorithm $[4,6]$ needs to store the frequent itemsets found in each level in main memory (it assumes that there is enough space) so that to create the candidate sets for next level. It needs so many passes through the data as the maximum size of a frequent itemset or two passes if we are only interested in frequent pairs as is the case in some applications. Improvements have been introduced in this original idea which address issues such as: if the main memory is not enough to accommodate counters for all pairs of items, then e.g., hashing is used to prune some infrequent pairs in the first pass.

In [21], the number of passes is reduced by taking a dynamic approach to the apriori algorithm which is called Dynamic Itemset Counting. It reduces the number of passes of apriori by starting counting 2-itemsets (and possibly 3-itemsets) during the first pass. After having read (say) one third of the data, it builds candidate 2-itemsets based on the frequent 1-itemsets count so far. Thus running on the rest two thirds of the data, it checks also the counts of these candidates and it stops checking the 2-itemsets counts during the second pass after having read the first third of data. Similarly, it may start considering 3-itemsets during the first pass after having read the first two thirds of the data and stops considering them during the second run. If the data is fairly homogeneous, this algorithm finds all frequent itemsets in around two passes.

In [89], a hash table is used to determine on the first pass (while the frequent items are being determined) that many pairs are not possibly frequent (assuming that there is enough main memory). The hash table is constructed so that each of its buckets stores the accumulative counts of more than one pairs. This algorithm works well when infrequent pairs have small counts so that even when all the counts of pairs in the same bucket are added, the result is still less than the threshold. In [44], multiple hash tables are used in the first pass and a candidate pair is required to be in a large bucket in every hash table. In the second pass another hash table is used to hash pairs and in the third pass, only if a pair belongs to a frequent bucket in pass two (and has passed the test of pass one too) is taken as a candidate pair. The multiple hash tables improve the algorithm when most of the buckets have counts a lot below the threshold (hence many buckets are likely to be small).

These methods, however, cannot be used for finding all frequent itemsets in one or two passes. Algorithms that find all frequent itemsets in one or two passes usually rely on randomness of data and sampling. A simple approach is to take a main-memorysized sample of the data, run one of the main-memory algorithms, find the frequent itemsets and either stop or run a pass through the data to verify. Some frequent itemsets might be missed in this way. In [94] this simple approach is taken. The algorithm on main memory is run on a much lower threshold so it is unlikely that it will miss a frequent itemset. To verify, we add to the candidates of the sample the negative border: an itemset $S$ is in the negative border if $S$ is not identified as frequent in the sample, but every immediate subset of $S$ is. The candidate itemsets includes all itemsets in the negative border. Thus the final pass through the data counts the frequency of the itemsets in the negative border. If no itemset in the negative border is frequent, then the sample has given all the frequent itemsets candidates. Otherwise, we may rerun the whole procedure if we do not want to miss any frequent itemset.

A large collection of algorithms have been developed for mining itemsets in vari- 
ous settings. Recent work in [71] provides a unifying approach for mining constrained itemsets, i.e., under a more general class of constraints than the minimuum support constraint. The approach is essentially a generalization of the a priori principle. Another consideration in this setting is that the collection of frequent itemsets found may be large and hard to visualize. Work done in [2] shows how to approximate the collection by a simpler bound without introducing many false positives and false negatives.

\subsection{Other Measures for Association Rules}

However confidence and support are not the only measures of "interestingness" of an association rule and do not always capture the intuition. Confidence is measured as the conditional probability of $X$ given $Y$ and it ignores the comparison to the (unconditional) probability of $X$. If the probability of $X$ is high then confidence might be measured above threshold although this would not imply any correlation among $X$ and $Y$. Other measures considered are the interest and the conviction [21]. Interest is defined as the probability of both $X$ and $Y$ divided by the product of the probability of $X$ times the probability of $Y$. It is symmetric with respect to $X$ and $Y$, and measures their correlation (or how far they are from being statistically independent). However, it can not derive an implication rule (which is non-symmetric). Conviction is defined as a measure closer to the intuition of an implication rule $X \Rightarrow Y$. Its motivation comes from the observation that if $X \Rightarrow Y$ is viewed as a logical implication, then it can be equivalently written as $\neg(X \wedge \neg Y)$. Thus conviction measures how far from statistical independence are the facts $X$ and $\neg Y$ and is defined as follows: $\frac{P(X) P(\neg Y)}{P(X, \neg Y)}$.

In [20] conditional probability is not used to measure interestingness of an association rule and propose statistical correlation instead. In [92], causal rules instead of mere associations are discussed aiming to capture the intuition whether $X \Rightarrow Y$ means that $X$ causes $Y$ or some other item causes them both to happen. This direction of investigation is taken by noticing that yielding a small number (possibly arbitrarily decided) of the "most interesting" causal relationships might be desirable in many data mining contexts, since exploratory analysis of a dataset is what is usually the aim of a data mining task. In that perspective, it is pointed out that ongoing research in Bayesian learning (where several techniques are developed to extract causal relationships) seems promising for large scale data mining.

\subsection{Mining for Similarity Rules}

As pointed out, various other kinds of rules may be of interest given a set of basket data. A similarity rule $X \simeq Y$ denotes that the itemsets $X$ and $Y$ are highly correlated, namely they are contained both in a large fraction of the transactions that contain either $X$ or $Y$. A similarity rule does not need to satisfy a threshold on the support, lowsupport rules are also of interest in this setting. Although for market basket analysis, the low support mining might not be very interesting, when the matrix represents the web graph, then similar web sites with low support might encompass similar subjects or mirror pages or plagiarism (in this case, rows will be sentences and columns web pages).

As low support rules are also of interest, techniques with support pruning (like finding all frequent itemsets) are not of use. However, in cases where the number of 
columns is sufficiently small then we can store something per column in main memory. A family of algorithms were developed in [34] to solve the problem in those cases using a hashing techniques.

For each column $C$, a signature $S(C)$ is defined which, intuitively, is a summary of the column. Signatures are such that a) they are small enough such that a signature for each column can fit in main memory and, b) similar columns have similar signatures. When the matrix is sparse, we cannot choose a small number of rows at random and use each shortened column in this set of rows as the signature. Most likely almost all signatures will be all 0's. The idea in this paper is: For each pair of columns, ignore the rows that both columns have zero entries, find the fraction of rows that these columns differ (over all non-both-zero-entry rows) and define this as the similarity measure. Interestingly, it can be proven that this similarity measure is proposrtional to the probability that both rows have the first occurrence of 1 in the same row. Thus the signature of each column is defined as the index of the first row with a 1 entry. Based on this similarity measure, two techniques that are developed in [34] are Min-Hashing (inspired by an idea in [33] -see also [24]) and Locality-Sensitive Hashing (inspired by ideas used in [56]-see also [65]).

In Min-Hashing, columns are hashed to the same bucket if they agree on the index of the first row with a 1 entry. To reduce the probability of false positives and false negatives, a set of $p$ signatures are collected instead of one signature. This is done by implicitly considering a set of $p$ different random permutations of the rows and for each permutation get a signature for each column. For each column, we use as its new signature the sequence of the $p$ row indices (the row where the first 1 entry appears in this column). Actually these $p$ row indices can be derived using only one pass through the data by hashing each row using $p$ different hash functions each hash function represents a permutation). However, if the number of columns is very large and we cannot afford work which is quadratic on the number of columns, then LocalitySensitive Hashing is proposed. Locality-Sensitive Hashing aims at reducing the number of pairs of columns that are to be considered by finding quickly many non-similar pairs of columns (and hence eliminating those pairs from further consideration). Briefly, it works as follows: It views the signatures in each column as a column of integers. It partitions the rows of this collection of rows into a number of bands. For each band it hashes the columns into buckets. A pair of columns is a candidate pair if they hash in the same bucket in any band. Tuning on the number of bands allows for a more efficient implementation of this approach.

If the input matrix is not sparse, a random collection of rows serves as a signature. Hamming LSH constructs a series of matrices, each with half as many rows as the previous, by OR-ing together two consecutive rows of the previous matrix.

These algorithms, although very efficient in practice, might still yield false positives and false negatives, i.e., yield a similarity rule which is false or miss some similarity rules. In [48], a family of algorithms is proposed which is called Dynamic Miss-Counting $(D M C)$ that avoid both false positives and false negatives. Two passes over the data are made and the amount of main memory used allows for data of moderate size. The key idea in DMC algorithms is confidence-pruning. For each pair of columns the algorithm counts the number of rows with entries in these columns that disagree and if the count exceeds a threshold they discard this similarity rule. 


\subsection{Transversals}

We point out here the connection between maximal frequent itemsets and transversals $[94,80]$ which are defined as follows: A hypergraph is a $0-1$ matrix with distinct rows. Each row can be viewed as an hyperedge and each column as an element. A transversal (a.k.a. hitting set) is a set of elements such that each hyperedge contains at least one element from the set. A transversal is minimal if no subset of it is a transversal.

Recall that a frequent itemset is a subset of the columns such that the number of rows with 1 entries in all those columns is above some support threshold. A maximal frequent itemset is a frequent itemset such that no superset is a frequent itemset. Given a support value, an itemset belongs to the negative border iff it is not a frequent itemset and all its subsets are frequent itemsets. The following proposition states the relationship between transversals and maximal frequent itemsets.

Proposition 5.1 Let $H_{F r}$ be the hypergraph of the complements of all maximal frequent itemsets, and let $H_{B d^{-}}$be the hypergraph of all itemsets in the negative border. Then the following holds:

1. The set of all minimal transversals of $H_{F r}$ is equal to the negative border.

2. The set of all minimal transversals of $H_{B d^{-}}$is equal to the set of all maximal itemsets.

It is not difficult to prove. A transversal $T$ of $H_{F r}$ has the property: For each maximal frequent itemset $S$, the transversal $T$ contains at least one attribute which is not included in this itemset $S$. Hence the transversal is not a maximal frequent itemset. Hence a minimal transversal belongs to the negative border.

For an example, suppose we have four attributes $\{A, B, C, D\}$ and let all maximal frequent itemsets be $\{\{A, B\},\{A, C\},\{D\}\}$, then the hypergraph of complements of those itemsets contains exactly the hyperedges $\{\{C, D\},\{B, D\},\{A, B, C\}\}$. All minimal transversals of this hypergraph are $\{\{C, B\},\{C, D\},\{A, D\},\{D, B\}\}$ which is equal to the negative border.

This result is useful because the negative border can be found easier in general and then used to retrieve the maximal frequent itemsets.

Transversals have been studied for a long time and hence this connection is useful. In [80] this result is extended in more general framework for which finding maximal frequent itemsets is a subcase. A connection is shown among the three problems of computing maximal frequent itemsets, computing hypergraph transversals and learning monotone boolean functions. This approach as well as the approach taken in [5] has its roots in the use of diagrams of models in model theory (see e.g., [30]).

For an excellent detailed exposition of algorithms mentioned in this section see [95].

\section{Clustering}

There are many different variants of the clustering problem and literature in this field spans a large variety of application areas and formal contexts. Clustering has many applications besides data mining including statistical data analysis, compression, vector 
quantization. It has been formulated in various contexts such as machine learning, pattern recognition, optimization and statistics. Several efficient heuristics have been invented. In this section, we will review some recent one pass algorithms and mention some considerations on the quality of clustering.

Informally, the clustering problem is that of grouping together (clustering) similar data items. One approach is to view clustering as a density estimation problem. We assume that in addition to the observed variables for each data item, there is a hidden, unobserved variable indicating the "cluster membership". The data is assumed to be produced by a model with hidden cluster identifiers. A mixture weight $w_{i}(x)$ is assumed for each data item $x$ to belong to a cluster $i$. The problem is estimating the parameters of each cluster $C_{i}, i=1, \ldots, k$, assuming the number $k$ of clusters is known. The clustering optimization problem is that of finding parameters for each $C_{i}$ which maximize the likelihood of the clustering given the model.

Most conventional clustering algorithms require space $\Omega\left(n^{2}\right)$ and require random access to the data. Hence recently several heuristics have been proposed for scaling clustering algorithms. Algorithms for clustering usually fall in two large categories $k$-median approach algorithms and hierarchical approach algorithms.

\subsection{The $k$-median approach}

A common formulation of clustering is the $k$-median problem: Find $k$ centers in a set of $n$ points so as to minimize the sum of distances from data points to their closest cluster centers. Or, equivalently, to minimize the average distance from data points to their closest cluster centers. The assumptions taken by the classical $k$-median approach are: 1) each cluster can be effectively modeled by a spherical Gaussian distribution, 2) each data item is assigned to one cluster and 3) the weights are assumed equal.

In [18], a single pass algorithm is presented for points in the Euclidean space and is evaluated by experiments. The method used is based in identifying regions of the data that are compressible (compression set), other regions that must be maintained in memory (retained set) and a third kind of regions that can be completely discarded (discard set). The discard set is set of points that are certain to belong to a specific cluster. They are discarded after they are used to compute the statistics of the cluster (such as the number of points, the sum of coordinates, the sum of squares of coordinates). The compression set is set of points that are close to each other so that it is certain that they will be assigned to the same cluster. They are replaced by their statistics (same as for the discard set). The rest of the points that do not belong in either of the two other categories remain in the retained set. The algorithm begins by storing a sample of points (the first points to be read) in main memory and running on them a main memory algorithm (such as $k$-means [66]). A set of clusters is obtained which will be modified as more points are read into main memory and processed. In each subsequent stage, a main-memory full set of points is processed as follows. 1. Determine if a set of points is (a) sufficiently close to some cluster $c_{i}$ and (b) unlikely for $c_{i}$ to "move" far from these points (during subsequent stages) and another cluster come closer. A discard set is decided in this way and its statistics used to update the statistics of the cluster. 2. Cluster the rest of the points in main memory and if a cluster is very tight, then replace the corresponding set of points by its statistics; this is a compression set. 3. Consider merging compression sets.

Similar summaries of data as in [18] and a data structure like an R-tree to store 
clusters are used in [50] to develop a one pass algorithm for clustering points in arbitrary metric spaces.

Algorithms with guaranteed performance bounds include a constant-factor approximation algorithm developed in [57] for the $k$-median problem. It uses a single pass on the data stream model and requires workspace $\theta\left(n^{\epsilon}\right)$ for a factor of $2^{O\left(\frac{1}{\epsilon}\right)}$. Other work includes [12] where the problem is studied on the sliding windows model.

A related problem is the $k$-center problem (minimize the maximum radius of a cluster) which is investigated in [32] where a single pass algorithm which requires workspace $O(k)$ is presented.

\subsection{The hierarchical approach}

A hierarchical clustering is a nested sequence of partitions of the data points. It starts with placing each point in a separate cluster and merges clusters until it obtains either a desirable number of clusters (usually the case) or a certain quality of clustering.

The algorithm CURE [58] handles large datasets and assumes points in Euclidean space. CURE employs a combination of random sampling and partitioning. A random sample, drawn from the data set, is first partitioned and cluster summaries are stored in memory in a tree data structure. For each successive data point, the tree is traversed to find the closest cluster to it.

Similarity measures according to which to cluster objects is also an issue of investigation. In [35], methods for determining similar regions in tabular data (given in a matrix) are developed. The proposed measure of similarity is based on the $L_{p}$ norm for various values for $p$ (non-integral too). It is noticed that on synthetic data, when clustering uses as a distance measure either $L_{1}$ or $L_{2}$ norms, the quality of the clustering is poorer than when $p$ is between 0.25 and 0.8 . The explanation for this is that, for large $p$, more emphasis is put on the outlier values (outliers are points that are isolated, so they do not belong to any cluster), whereas for small $p$ the measure approaches the Hamming distance, i.e., it counts how many values are different. On real data, it is noticed that different values for $p$ bring out different features of the data. Therefore, it seems that $p$ can be used as a useful parameter of the clustering algorithm: set $p$ higher to show full details of the data set, reduce $p$ to bring out unusual clusters in the data. For the technical details to go through, sketching techniques similar to [62] are used to approximate the distances between subtables and reduce the computation. The proposed similarity measure is tested using the $k$-means algorithm to cluster tabular data.

\subsection{Clustering documents by Latent Semantic Indexing (LSI)}

The use of vector space models for information retrieval purposes has been used as early as 1957. The application of SVD in information retrieval is proposed in [37] through the latent semantic indexing technique and it is proven a powerful approach for dimension reduction. The input matrix $\vec{X}$, is a document versus terms matrix. It could be a 0,1-matrix or each entry could be the frequency of the term in this document. 
Matrix $\vec{X}$ is approximated according to SVD by $\vec{X}_{k}=\vec{U}_{k} \vec{T}_{k} \vec{V}_{k}^{T r}$. The choice of $k$ is an issue for investigation. Note that each entry of the matrix $\vec{X}_{k}$ does not correspond to a term any more, it corresponds to a weighted sum of term measures. The matrix $V_{k}$ represents similarities among documents, e.g., given a document that the user is interested in more documents can be decided that are of interest to this user (even if they do not use exactly the same terms). The matrix $U_{k}$ displays similarities between terms, e.g., given a term, other related terms may be decided (such the term "car" is related to "automobile" and "vehicle"). The matrix $X_{k}$ may be used for term-document associations, e.g., on a given term, extract documents that contain material related to this term.

Spectral methods -i.e., the use of eigenvectors and singular vectors of matrices-in document information retrieval and the application of SVD through the latent semantic indexing technique are discussed in detail in [73], which is an excellent survey on this direction of research.

\subsection{Quality of Clustering}

In a clustering algorithm the objective is to find a good clustering but a good clustering is not formally defined. Intuitively the quality of a clustering is assessed by how much similar points are grouped in the same cluster. In [68] the question is posed: how good is the clustering which is produced by a clustering algorithm? As already discussed the $k$-median clustering may produce a very bad clustering in case the "hidden" clusters are far from spherical. E.g., imagine two clusters, one that is a sphere and a second one is formed at a certain distance around the sphere forming a ring. Naturally the $k$-median approach will fail to produce a good clustering in this example. A bicriteria measure is proposed therein for assessing the quality of clusters. The dataset is represented as a graph with weights on the edges that represent the degree of similarity between the two vertices (high weight means high similarity). First a quantity which measures the relative minimum cut of a cluster is defined. It is called expansion and is defined as the weight of the minimum cut divided by the number of points in the smaller subset among the two that the cut partitions the cluster-graph. It seems however that it is more appropriate to give more importance to vertices with many similar other vertices than to vertices with few similar other vertices. Thus, the definition is extended to capture this observation and the conductance is defined where subsets of vertices are weighted to reflect their importance. Optimizing the conductance gives the right clustering in the sphere-ring example. However if we assume the conductance as the measure of quality, then imagine a situation where there are mostly clusters of very good quality and a few points that create clusters of poor quality. In this case the algorithm might create many smaller clusters of medium quality. A second criterion is considered in order to overcome this problem. This criterion is defined as the fraction of the total weight of edges that are not covered by any cluster.

This bicriterion optimization framework is used to measure the quality of several spectral algorithms. These algorithms, though have proven very good in practice, were hitherto lacking a formal analysis. 


\subsection{Bibliographical Notes}

Popular clustering algorithms in the literature include $k$-means [66], CLARANS [83], BIRCH [100], DBSCAN [41].

In [86], results from [57] are used to develop an algorithm that achieves dramatically better clustering quality than BIRCH although it takes longer to run. In [3], they define clusters in euclidean space by DNF formulas and address performance issues for data mining applications. In [87], the drawbacks of random sampling in clustering algorithms (e.g., small clusters might be missed) are avoided by density biased sampling. The goal is to under-sample dense regions and over-sample sparse regions of the data. A memory efficient single pass algorithm is proposed that approximates density biased sampling.

An excellent detailed exposition of algorithms in [58], [18] and [50] can be found in [95]. An excellent survey of the algorithms in [55, 3, 13, 15] is given in [45].

\section{Mining the web}

The challenge in mining the web for useful information is the huge size and unstructured organization of data. Search engines, one of the most popular web mining applications, aim to search the web for a specific topic and give to the user the most important web pages on this topic. A considerable amount of research has been done on ranking web pages according to their importance.

Page Rank, the algorithm used by the Google search engine [22] ranks pages according to the page citation importance. This algorithm is based on the observation that usually important pages have many other important pages linking to them. Thus it is an iterative procedure which essentially computes the principal eigenvector of a matrix. The matrix has one non zero entry for each link from page $i$ to page $j$ and this entry is equal to $1 / n$ if page $i$ has $n$ successors (i.e., links to other pages). The intuition behind this algorithm is that page $i$ shares its importance among its successors. Several variants of this algorithm have been developed to solve problems concerning spams and dead ends (pages with no successors). Random jumps from a web page to another may be used to avoid dead ends or a slight modification of the iterative procedure according to which some of the importance is equally distributed among all pages in the beginning.

Hubs and Authorities, based on similar intuition, are viewed also as sharing their importance to its successors only that there are two different roles assigned to important web pages [72]. They follow the observation that authorities might not link to one another directly but there are hubs that link "collectively" to many authorities. Thus hubs and authorities have this mutually depending relationship: good hubs link to many authorities and good authorities are linked by many hubs. Hubs are web pages that do not contain information themselves but they contain many links to pages with information e.g., a university course homepage. Authorities are pages that contain information about a topic, e.g., a research project homepage. Again the algorithm based on this idea is an iterative procedure which computes eigenvectors of certain matrices. It begins with matrix $A$ similar as the page rank algorithm only that the entries are either 0 or 1 (if there is a link) and its output is two vectors which measure the "authority" and the "hubbiness" of each page. These vectors are the principal 
eigenvectors of the matrices $A A^{T}$ and $A^{T} A$. Work in $[54,77]$ has shown that the concepts of hubs and authorities is a fundamental structural feature of the web. The CLEVER system [29] builds on the algorithmic framework of hub and authorities.

Other work on measuring the importance of web pages include [1]. Other research directions for mining useful information from the web include [23], where the web is searched for frequent itemsets by a method using features of the algorithm for dynamic itemset counting [21]. Instead of a single deterministic run, the algorithm runs continuously exploring more and more sites. In [19], the extraction of structured data is achieved from information offered by unstructured data on the web. The example used is to search for books in the web starting from a small sample of books from which a pattern is extracted. Based on the extracted patterns more books are retrieved in a iterative manner. Based on the same idea of pattern matching, the algorithm developed in [9] searches the web for communities that share an interest on a topic. The pattern is formed by using words from the anchor text.

More detailed descriptions of the Page Rank and the Hubs and Authorities algorithms can be found in [95]. Also, an elegant formal exposition of spectral methods used for web mining and the connections between this work and earlier work on sociology and citation analysis [39] can be found in [73].

\section{Evaluating the results of data mining}

As we have seen, for many of the successful data mining algorithms there is no formal analysis as to whether the solution they produce is a good approximation to the problem at hand. Recently a considerable amount of research is focused in developing criteria for such an evaluation.

A line of research is focused in building models for practical situations (like the link structure of the web or a corpus of technical documents) against which to evaluate algorithms. Naturally, the models, in order to be realistic, are shown to display several of the relevant features that are measured in real situations (e.g., the distribution of the number of outgoing links from a web page).

In [88], a model for documents is developed on which the LSI method is evaluated. In this model, each document is built out of a number of different topics (hidden from the retrieval algorithm). A document on a given topic is generated by repeating a number of times terms related to the topic according to a probability distribution over the terms. For any two different topics there is a technical condition on the distributions that keeps the topics "well-separated". The main result is that on this model, the $k$-dimensional subspace produced by LSI defines with high probability very good clusters as intended by the hidden parameters of the model. In this paper, it is also proposed that if the dimensionality after applying LSI is too large, then random projection can be used to reduce it and improve the results. Other work with results suggesting methods for evaluating spectral algorithms include [10].

Models for the web graph are also developed. The web can be viewed as a graph with each page being a vertex and an edge exists if there is a link pointing from one web page to another. Measurements on the web graph [76, 77] have shown that this graph has several characteristic features which play a major role in the efficiency of several known algorithms for searching the web. In that context, the web graph is a power-law graph, which means roughly that the probability that a degree is larger than 
$d$ is at least $d^{-\beta}$ for some $\beta>0$. Models for power-law graphs are developed in [42],[7], [78].

A technique for automatically evaluating strategies which find similar pages on the web is presented in [60]. A framework for evaluating the results of data mining operations according to the utility of the results in decision making is formalized in [74] as an economically motivated optimization problem. This framework leads to interesting optimization problems such as the segmentation problem which is studied in [75]. Segmentation problems are related to clustering.

\section{Conclusion}

We surveyed some of the formal techniques and tools for solving problems on the data stream model and on similar models where there are constraints on the amount of main memory used and a few number of passes through the data are allowed because access is too costly. We also presented some of the most popular algorithms that are proposed in the literature for data mining applications. We provided reference for further reading, with some good surveys and tutorials in the end of each section. As the field is a rapidly evolving area of research with many diverging applications, the exposition here is meant to serve as an introduction to approximation algorithms with storage constraints and their applications.

Among topics that we did not mention are Privacy preserving data mining, Time Series Analysis, Visualization of Data Mining results, Bio-informatics, Semistructured data and $X M L$.

Acknowledgements: Thanks to Chen Li and Ioannis Milis for reading and providing comments in an earlier version of this chapter.

\section{References}

[1] S. Abiteboul, M. Preda, and G. Cobena. Adaptive on-line page importance computation. In $V L D B, 2002$.

[2] F. Afrati, A. Gionis, and H. Mannila. Approximating a collection of frequent sets. In $K D D, 2004$.

[3] R. Agrawal, J. Gehrke, D. Gunopulos, and P. Raghavan. Automatic subspace clustering of high dimensional data for data mining applications. In SIGMOD, 1998.

[4] R. Agrawal, T. Imielinski, and A. Swami. Mining associations between sets of items in massive databases. In SIGMOD, pages 207-216, 1993.

[5] R. Agrawal, H. Mannila, R. Srikant, H. Toivonen, and A. I. Verkamo. Fast discovery of association rules. In U. Fayyad, G. Piatetsky-Shapiro, P. Smyth, and R. Uthurusamy, editors, Advances in Knowledge Discovery and Data Mining. 1996.

[6] R. Agrawal and R. Srikant. Fast algorithms for mining association rules. In $V L D B, 1994$.

[7] W. Aiello, F. Chung, and L. Lu. A random graph model for power law graphs. In $S T O C, 2000$.

[8] N. Alon, Y. Matias, and M. Szegedy. The space complexity of approximating frequency moments. In STOC, pages 20-29, 1996. 
[9] N. AlSaid, T. Argyros, C. Ermopoulos, and V. Paulaki. Extracting cyber communities through patterns. In SDM, 2003.

[10] Y. Azar, A. Fiat, A. Karlin, F. McSherry, and J. Saia. Spectral analysis of data. In $S T O C$, pages 619-636, 2001.

[11] B. Babcock, S. Babu, M. Datar, R. Motwani, and J. Widom. Models and issues in data stream systems. In PODS, 2002.

$[12$ B. Babcock, M. Datar, R. Motwani, and L. O'Callaghan.

13] J. Banfield and A. Raftery. Model-based gaussian and non-gaussian clustering. Biometrics, 49:803-821, 1993.

[14] Z. Bar-Yossef, R. Kumar, and D. Sivakumar. Sampling algorithms: Lower bounds and applications. In STOC, 2001.

[15] A. Ben-Dor and Z. Yakhini. Clustering gene expression patterns. In RECOMB, 1999.

[16] J. Berger. Statistical Decision Theory and Bayesian Analysis. Springer Verlag, 1985.

[17] A. Borodin, R. Ostrovsky, and Y. Rabani. Subquadratic approximation algorithms for clustering problems in high dimensional spaces. In STOC, 1999.

[18] P. Bradley, U. Fayyad, and C. Reina. Scaling clustering algorithms to large databases. In $K D D, 1998$.

[19] S. Brin. Extracting patterns and relations from the world-wide web., 1998.

[20] S. Brin, R. Motwani, and C. Silverstein. Beyond market baskets: Generalizing association rules to correlations. In SIGMOD, pages 265-276, 1997.

[21] S. Brin, R. Motwani, J. D. Ullman, and S. Tsur. Dynamic itemset counting and implication rules for market basket data. In SIGMOD, pages 255-264, 1997.

[22] S. Brin and L. Page. The anatomy of a large-scale hypertextual web search engine. In WWWr/Computer Networks, pages 107-117, 1998.

[23] S. Brin and L. Page. Dynamic data mining: Exploring large rule space by sampling., 1998.

[24] A. Broder. On the resemblance and containment of documents. In Compression and Complexity of Sequences, pages 21-29, 1997.

[25] A. Broder, M. Charikar, A. Frieze, and M. Mitzenmacher. Min-wise independent permutations. In STOC, 1998.

[26] A. Broder, S. Glassman, M. Manasse, and G. Zweig. Syntactic clustering of the web. In Sixth International World Wide We Conference, pages 391-404, 1997.

[27] H. Buhrman and R. de Wolf. Complexity measures and decision tree complexity: A survey. available at: http://www.cwi.nl/ rdewolf, 1999.

[28] R. Canetti, G. Even, and O. Goldreich. Lower bounds for sampling algorithms for estimating the average. Information Processing Letters, 53:17-25, 1995.

[29] S. Chakrabarti, B. Dom, R. Kumar, S. R. P. Raghavan, and A. Tomkins. Experiments in topic distillation. In SIGIR workshop on hypertext information retrieval, 1998.

[30] C. Chang and H. Keisler. Model Theory. North Holland, Amsterdam, 1990.

31] M. Charikar, S. Chaudhuri, R. Motwani, and V. Narasayya. Towards estimation error guarantees for distinct values. In PODS, pages 268-279, 2000.

[32] M. Charikar, C. Chekuri, T. Feder, and R. Motwani. Incremental clustering and dynamic information retrieval. In STOC, pages 626-635, 1997.

[33] E. Cohen. Size-estimation framework with applications to transitive closure and reachability. Journal of Computer and Systems Sciences, 55:441-453, 1997.

[34] E. Cohen, M. Datar, S. Fujiwara, A. Gionis, P. Indyk, R. Motwani, J. D. Ullman, and C. Yang. Finding interesting associations without support pruning. In TKDE 13(1) 2001 and also in ICDE, pages 64-78, 2000.

[35] G. Cormode, P. Indyk, N. Koudas, and S. Muthukrishnan. Fast mining of massive tabular data via approximate distance computations. In ICDE, 2002. 
[36] P. Dagum, R. Karp, M. Luby, and S. Ross. An optimal algorithm for monte carlo estimation. In FOCS, pages 142-149, 1995.

[37] S. Deerwester, S. Dumais, G. Furnas, T. Landauer, and R. Harshman. Indexing by latent semantic analysis. The American Society for Information Science, 41(6):391-407, 1990.

[38] A. Dobra, M. Garofalakis, and J. Gehrke. Sketch-based multi-query processing over data streams. In EDBT, 2004.

[39] L. Egghe and R. Rousseau. Introduction to Informetrics. 1990.

[40] L. Engebretsen, P. Indyk, and R. O'Donnell. Derandomized dimensionality reduction with applications. In SODA, 2002.

[41] M. Ester, H.-P. Kriegel, J. Sander, and X. Xu. A density-based algorithm for discovering clusters in large spatial databases with noise. In Second International Conference on Knoweledge Discovery and Data Mining, page 226, 1996.

[42] A. Fabrikant, E. Koutsoupias, and C. H. Papadimitriou. Heuristically optimized trade-offs: A new paradigm for power laws in the internet. In STOC, 2002.

[43] C. Faloutsos. Indexing and mining streams. In SIGMOD, 2004.

[44] M. Fang, N. Shivakumar, H. Garcia-Molina, R. Motwani, and J. Ullman. Computing iceberg queries efficiently. In $V L D B, 1998$.

[45] D. Fasulo. An analysis of recent work on approximation algorithms. Technical Report 01-03-02, University of Washington, Dept. of Computer science and Engineering, 1999.

[46] J. Feigenbaum, S. Kannan, M. Strauss, and M. Viswanathan. An approximate 11-difference for massive data streams. In FOCS, 1999.

[47] W. Feller. An Introduction to Probability Theory and Its Applications. John Wiley, New York, 1968.

[48] S. Fujiwara, J. D. Ullman, and R. Motwani. Dynamic miss-counting algorithms: Finding implication and similarity rules with confidence pruning. In $I C D E$, pages 501-511, 2000.

[49] S. Gangulya, M. Garofalakis, and R. Rastogi. Sketch-based processing data streams join aggregates using skimmed sketches. In EDBT, 2004.

[50] V. Ganti, R. Ramakrishnan, J. Gehrke, A. L. Powell, and J. C. French. Clustering large datasets in arbitrary metric spaces. In ICDE, pages 502-511, 1999.

[51] M. Garofalakis, J. Gehrke, and R. Rastogi. Querying and mining data streams: You only get one look. In $V L D B, 2002$, also available at: http://www.belllabs.com $/^{\sim}$ minos.

[52] P. Gibbons and Y. Matias. Synopsis data structures for massive data sets. In SODA, pages S909-S910, 1999.

[53] P. Gibbons and S. Tirthapura. Estimating simple functions on the union of data streams. In ACM Symposium on Parallel Algorithms and Architectures, pages 281-291, 2001.

[54] D. Gibson, , and P. R. Jon M. Kleinberg. Inferring web communities from link topology. In ACM Conference on Hypertext and Hypermedia, volume 8(3-4), 1998.

[55] D. Gibson, J. M. Kleinberg, and P. Raghavan. Two algorithms for nearest neighbor search in high dimensions. In STOC, volume 8(3-4), 1997.

[56] A. Gionis, P. Indyk, and R. Motwani. Similarity search in high dimensions via hashing. In $V L D B$, pages 518-529, 1999.

[57] S. Guha, N. Mishra, R. Motwani, and L. O'Callaghan. Clustering data streams. In FOCS, 2000.

[58] S. Guha, R. Rastogi, and K. Shim. Cure: An efficient clustering algorithm for large databases. In SIGMOD, 1998. 
[59] D. Hand, H. Mannila, and P. Smyth. Principles of Data Mining (Adaptive computation and machine learning). MIT Press, 2001.

[60] T. Haveliwala, A. Gionis, D. Klein, and P. Indyk. Similarity search on the web: Evaluation and scalable considerations. In 11th International World Wide Web Conference, 2002.

[61] M. R. Henzinger, P. Raghavan, and S. Rajagopalan. Computing on data streams. available at: http://www.research.digital.com/SRC/, 1998.

[62] P. Indyk. Stable distributions, pseudorandom generators, embeddings and data stream computation. In FOCS, pages 189-197, 2000.

[63] P. Indyk. Algorithmic applications of low-distortion geometric embeddings. In FOCS, 2001.

[64] P. Indyk, N. Koudas, and S. Muthukrishnan. Identifying representative trends in massive time series data sets using sketches. In $V L D B$, pages 363-372, 2000.

[65] P. Indyk and R. Motwani. Approximate nearest neighbors: Towards removing the curse of dimensionality. In STOC, pages 604-613, 1998.

[66] A. Jain and R. Dubes. Algorithms for Clustering Data. Prentice Hall, 1988.

[67] W. Johnson and J. Lindenstrauss. Extensions of lipschitz mapping into hilbert space. Contemporary Mathematics, 26:189-206, 1984.

[68] R. Kannan, S. Vempala, and A. Vetta. On clusterings - good, bad and spectral. In FOCS, pages 367-377, 2000.

[69] A. Karlin, M. Manasse, L. Rodolph, and D. Sleator. Competitive snoopy caching. In $S T O C$, pages 70-119, 1988.

[70] M. Kearns and U. Vazirani. An introduction to comoputational learning theory. MIT Press, 1994.

[71] D. Kifer, J. Gehrke, C. Bucila, and W. White. How to quickly find a witness. In PODS, 2003.

[72] J. Kleinberg. Authoritative sources in a hyperlinked environment. J.ACM, 46(5):604-632, 1999.

[73] J. Kleinberg and A. Tomkins. Applications of linear algebra in information retrieval and hypertext analysis. In PODS, pages 185-193, 1999.

[74] J. M. Kleinberg, C. H. Papadimitriou, and P. Raghavan. A microeconomic view of data mining. Data Mining and Knowledge Discovery, 2(4):311-324, 1998.

[75] J. M. Kleinberg, C. H. Papadimitriou, and P. Raghavan. Segmentation problems. In $S T O C$, pages $473-482,1998$.

[76] S. Kumar, P. Raghavan, S. Rajagopalan, R. Stata, A. Tomkins, and J. Wiener. Graph structure in the web: experiments and models. In International World Wide Web Conference, pages 309-320, 2000.

[77] S. Kumar, P. Raghavan, S. Rajagopalan, and A. Tomkins. Trawling emerging cybercommunities automatically. In International World Wide Web Conference, volume 8(3-4), 1999.

[78] S. Kumar, P. Raghavan, S. Rajagopalan, A. Tomkins, and E. Upfal. Stochastic models for the web graph. In FOCS, pages 57-65, 2000.

[79] E. Kushilevitz and N. Nisan. Communication Complexity. Cambridge University Press, 1997.

[80] H. Mannila and H. Toivonen. On an algorithm for finding all interesting sentences. In Cybernetics and Systems, Volume II, The Thirteenth European Meeting on Cybernetics and Systems Research, pages 973 - 978, 1996.

[81] R. Motwani and P. Raghavan. Randomized Algorithms. Cambridge University Press, 1995.

[82] J. Munro and M. Paterson. Selection and sorting with limited storage. Theoretical Computer Science, 12:315-323, 1980. 
[83] R. Ng and J. Han. Efficient and effective clustering methods for spatial data mining. In $V L D B$, pages 144-155, 1994.

[84] N. Nisan. Pseudorandom generators for pseudorandom computations. In STOC, pages 204-212, 1990.

[85] J. Nolan. An introduction to stable distributions. http://www.cas.american.edu/ jpnolan/chap1.ps.

[86] L. O'Callaghan, N. Mishra, A. Meyerson, S. Guha, and R. Motwani. Streamingdata algorithms for high-quality clustering. In ICDE, 2002.

[87] C. Palmer and C. Faloutsos. Density biased sampling: An improved method for data mining and clustering. In SIGMOD, pages 82-92, 2000.

[88] C. H. Papadimitriou, P. Raghavan, H. Tamaki, and S. Vempala. Latent semantic indexing: A probabilistic analysis. JCSS, 61(2):217-235, 2000.

[89] J. S. Park, M.-S. Chen, and P. S. Yu. An effective hash-based algorithm for mining association rules. In SIGMOD, pages 175-186, 1995.

[90] M. Saks and X. Sun. Space lower bounds for distance approximation in the data stream model. In STOC, 2002.

[91] L. Schulman and V. Vazirani. Majorizing estimators and the approximation of $\sharp \mathrm{p}$-complete problems. In STOC, pages 288-294, 1999.

[92] C. Silverstein, S. Brin, R. Motwani, and J. D. Ullman. Scalable techniques for mining causal structures. In Data Mining and Knowledge Discovery 4(2/3), pages 163-192, 2000.

[93] STREAM. Stanford stream data management project. http://wwwdb.stanford.edu/stream.

[94] H. Toivonen. Sampling large databases for association rules. In $V L D B$, pages 134-145, 1996.

[95] J. Ullman. Lecture notes on data mining. available at: http://wwwdb.stanford.edu/ ullman/cs345-notes.html, 2000.

[96] V. Vapnik. Statistical learning theory. John Wiley, 1998.

[97] V. Vazirani. Approximation algorithms. Springer, 2001.

[98] D. Vengroff and J. Vitter. I/o efficient algorithms and environments. Computing Surveys, page 212, 1996.

[99] J. Vitter. Random sampling with a reservoir. ACM Trans. on Mathematical Software, 11(1):37-57, 1985.

[100] T. Zhang, R. Ramakrishnan, and M. Livny. Birch: An efficient data clustering method for very large databases. In SIGMOD, pages 103-114, 1996. 